\title{
Rapid Reversible Changes in Dendritic Spine Structure In Vivo Gated by the Degree of Ischemia
}

\author{
Shengxiang Zhang, ${ }^{1,2}$ Jamie Boyd, ${ }^{4}$ Kerry Delaney, ${ }^{4}$ and Timothy H. Murphy ${ }^{1,2,3}$ \\ ${ }^{1}$ Kinsmen Laboratory, Department of Psychiatry, ${ }^{2}$ Brain Research Center, and ${ }^{3}$ Department of Cellular and Physiological Sciences, University of British \\ Columbia, Vancouver, British Columbia, Canada V6T 1Z3, and ${ }^{4}$ Department of Biology, University of Victoria, Victoria, British Columbia, Canada V8W 3N5
}

Current therapeutic windows for effective application of thrombolytic agents are within 3- $6 \mathrm{~h}$ of stroke. Although treatment can improve outcome, it is unclear what happens to synaptic fine structure during this critical period in vivo. The relationship between microcirculation and dendritic spine structure was determined in mouse somatosensory neurons during stroke. Spines were, on average, $13 \mu \mathrm{m}$ from a capillary and were supplied by $\sim 100$ red blood cells per second. Moderate ischemia ( $\sim 50 \%$ supply) did not significantly affect spines within $5 \mathrm{~h}$; however, severe ischemia ( $<10 \%$ supply) caused a rapid loss of spine and dendrite structure within as little as $10 \mathrm{~min}$. Surprisingly, if reperfusion occurred within 20-60 min, dendrite and spine structure was mostly restored. These data suggest that the basic dendritic wiring diagram remains mostly intact during moderate ischemia and that affected synapses could potentially contribute to functional recovery. With severe ischemia, markedly deformed dendritic structure can partially recover if reperfusion occurs early.

Key words: stroke; dendritic spine; plasticity; fluorescence microscopy; cerebral blood flow; dendritic arborization; glutamatergic neurons; ischemia; PSD; recovery; structural plasticity

\section{Introduction}

In adult animals, under basal conditions, dendritic spines are relatively stable and exhibit turnover times on the order of days to weeks (Grutzendler et al., 2002; Trachtenberg et al., 2002; Holtmaat et al., 2005). However, it is unclear whether the stability of mature spines can be extended to conditions that challenge the integrity of the nervous system in vivo, such as ischemic stroke. Associated with ischemia are metabolic compromise and aberrant activation of signal transduction pathways leading to neuronal death (Barber et al., 2003). These pathways include the massive overstimulation of ionotropic and metabotropic glutamate receptors. Conceivably, neurotransmitter and other secondmessenger pathways that regulate changes in dendritic spine motility and function during synaptogenesis (Hering and Sheng, 2001; Bonhoeffer and Yuste, 2002) may be aberrantly stimulated during ischemia, resulting in altered spine turnover or structural derangement, which could potentially impact stroke recovery. Accordingly, we examined the relationship between blood flow and dendritic spine structure in vivo during either moderate or severe ischemia using repeated two-photon imaging in mice expressing yellow fluorescent protein (YFP) in a select subpopulation of layer 5 somatosensory neurons (Feng et al., 2000). Re-

Received March 20, 2005; revised April 18, 2005; accepted April 21, 2005.

This work was supported by Operating Grants MOP49586 (T.H.M.) and MOP14455 (K.D.) from the Canadian Institutes of Health Research and the Canadian Stroke Network. T.H.M. and K.D. are Michael Smith Foundation for Health Research Senior Scholars. We thank Ping Li and Lei Jiang for technical support and A. Shih and X. Sun for assistance with preliminary experiments.

Correspondence should be addressed to Dr. Timothy H. Murphy, Department of Psychiatry, University of British Columbia, 4N1-2255 Wesbrook Mall, Vancouver, British Columbia, Canada V6T 1Z3. E-mail: thmurphy@interchange.ubc.ca.

DOI:10.1523/JNEUROSCI.1085-05.2005

Copyright $\odot 2005$ Society for Neuroscience $\quad$ 0270-6474/05/255333-06\$15.00/0 peated image acquisition on the same animal has advantages over single time point histological studies, in that relatively subtle changes in spine number, shape, or position can be observed because the same synapses are sampled repeatedly. It is likely that such changes will be undetected in histological studies after stroke in which comparisons are made between animals (Biernaskie and Corbett, 2001; Kolb et al., 2003).

By imaging both microcirculation and dendrites, we report that dendrite and spine structure are relatively resistant to moderate ischemia. However, severe ischemia was associated with widespread loss of dendritic structure within minutes. Interestingly, from a therapeutic standpoint, if reperfusion occurred within 20-60 min, the structural changes were in part reversible. These data suggest that major structural changes to synapses are occurring within the ischemic core during human stroke. If therapeutic intervention can stimulate reperfusion promptly, these changes may be partially reversible, allowing affected synaptic networks to contribute to the recovery process.

\section{Materials and Methods}

Animal preparation. C57BL/6 transgenic mice (H-line) expressing YFP in a subset of layer 5 cortical neurons (Feng et al., 2000) were purchased from The Jackson Laboratory (Bar Harbor, ME) and bred at the University of British Columbia animal facilities. A total of 37 transgenic and 13 wild-type mice (of both sexes) were used in the study. Protocols were approved by the animal care committee, consistent with Canadian Council on Animal Care and Use guidelines. Mice aged 3-5 months were deeply anesthetized with an intraperitoneal injection of urethane $(0.12 \%$ $\mathrm{w} / \mathrm{w}$, supplemented at $0.02 \% \mathrm{w} / \mathrm{w}$ as needed) (Kleinfeld et al., 1998) and supplemented with $20 \mathrm{~mm}$ glucose in PBS (0.2-0.3 ml, i.p., every $1 \mathrm{~h}$ ). Body temperature was maintained at $37^{\circ} \mathrm{C}$ using a heating pad. A $2 \times 2$ $\mathrm{mm}^{2}$ cranial window was made over the somatosensory cortex at coordinates of $-0.8 \mathrm{~mm}$ from bregma and $2.0 \mathrm{~mm}$ lateral, leaving the dura 
intact. A stainless-steel chamber that surrounded the craniotomy was glued to the skull with Krazy Glue (Elmer's Products, Columbus, $\mathrm{OH})$. To reduce movement artifacts, the area between the chamber and the skull was filled with dental acrylic (Kleinfeld and Denk, 2000). The exposed cortical surface and chamber were filled with $2 \%(\mathrm{w} / \mathrm{v})$ agarose (diluted in PBS or a HEPES-buffered artificial CSF) and sealed by a cover glass (\#1). To image blood flow, blood plasma was labeled through a tail-vein injection of a $0.1 \mathrm{ml}$ bolus of $5 \%(\mathrm{w} / \mathrm{v})$ fluorescein isothiocyanate (FITC)-labeled dextran $(500 \mathrm{kDa})$ or Texas Red dextran $(70 \mathrm{kDa})$ in PBS.

Intracortical microinjection for endothelin-1 model. The imaging chamber was opened, the agarose was removed, and $2 \mu \mathrm{l}$ of endothelin-1 (ET-1) $(1 \mu \mathrm{g} / \mu \mathrm{l})$ or PBS was injected $\sim 0.5 \mathrm{~mm}$ from the site of imaging at a depth of $0.5 \mathrm{~mm}$ over a $30 \mathrm{~min}$ period (using 60 - to $90-\mu \mathrm{m}$ diameter glass pipettes). On completion of the injection, the chamber was refilled with agarose and sealed with a coverslip. The animal was then returned to the two-photon microscope, and the original imaging volume was found using vascular landmarks. Laser Doppler measurements of blood flow were made from wildtype C57BL/6 mice through the skull using a Perimed (Piscataway, NJ) PF3 with a 1-mmdiameter probe.

Rose bengal cortical photothrombosis model. To apply rose bengal (RB), we injected the dye $(0.03 \mathrm{mg} / \mathrm{g}$ mouse, diluted to $10 \mathrm{mg} / \mathrm{ml}$ in PBS) into the tail vein. Photoactivation was performed by exposing the cortex continuously to green light ( $535 \pm 25 \mathrm{~nm}$ ) for $1-2$ min using a $10 \times 0.3$ numerical aperture objective to activate the dye over an $\sim 2 \mathrm{~mm}^{2}$ area (the average power through the objective was $\sim 3 \mathrm{~mW}$ ).

Imaging. Mice were maintained under urethane anesthesia for the entire imaging session.

Animals were fitted into a custom-made two-photon microscope. Two-photon fluorescence excitation was performed with a Coherent (Santa Clara, CA) Mira 900 Ti:sapphire laser pumped by a $5 \mathrm{~W}$ Verdi laser and was tuned to $920 \mathrm{~nm}$ to excite YFP. Images were acquired using custom software routines (IgorPro; Wavemetrics, Eugene, OR) and by using an Olympus (Tokyo, Japan) IR-LUMPlanFl water-immersion objective $(60 \times ; 0.9$ numerical aperture).

For in vivo time-lapse imaging of dendritic structure, multiple $Z$-series were taken at the indicated time intervals over $5 \mathrm{~h}$. The spacing of successive $Z$-images was $1.0 \mu \mathrm{m}$ and provided sufficient overlap between sections when the axial resolution of the imaging system at the settings used was $\sim 2.0 \mu \mathrm{m}$. The imaged dendrites were typically within $100 \mu \mathrm{m}$ of the pial surface and therefore were in layer 1 . The images were acquired with a pixel size of $0.13 \mu \mathrm{m}$, typically spanning a $140 \times 140 \mu \mathrm{m}$ area, and were the average of three frames.

For histology, animals were overdosed with anesthetic and transcardially perfused with PBS, followed by ice-cold $4 \%$ paraformaldehyde in PBS. Frozen sections $(20 \mu \mathrm{m})$ were stained with Fluorojade (Histochem, Jefferson, AR).

Image analysis. Image analysis was performed using NIH ImageJ software (http://rsb.info.nih.gov/ij/). To reduce photon and photomultiplier tube noise, a median filter (radius, 2) was applied. Spine counting was done with the evaluator blinded to the experimental condition. The velocity of red blood cells (RBCs) was measured from repetitive line scanning along the central axis of a capillary (Kleinfeld et al., 1998). To esti- mate RBC velocity, we fit a line to the arcs created by moving RBCs in line scans and calculated a change in position versus time $(\Delta x / \Delta t)$. The RBC supply rate was determined by counting the number of RBCs that passed a given point in the capillary, measured by counting the number of bands on a line scan that intersect a line perpendicular to the scan direction. We typically performed three scans for two to three different cortical depths $(0.27 \mu \mathrm{m} /$ pixel and $1.14 \mathrm{~ms} /$ line; 512 lines per scan) for each time point. To assess spine turnover, we made two-dimensional maximal intensity projections over $5-15 \mu \mathrm{m}$ regions to isolate dendritic branches of the tuft that projected laterally. Considering the relatively poor resolution of the microscope in the axial direction, we only analyzed spines that projected mainly parallel to the imaging plane. Images from different time points were aligned and compared. Spines were considered to disappear if they no longer constituted a visually distinct structure extending from the parent dendrite. Visually distinct means that an area with reduced brightness indicative of a spine neck could be observed. In cases in which spines had short necks, evidence for a rounded head (more than a half circle) was used as a criterion to distinguish between dendritic swellings and spines. $Z$-stacks were used to ensure that tissue distortion caused by stroke between different time points did not influence spine identification. The resolution of two-photon microscopy in vivo is limited by a 
a

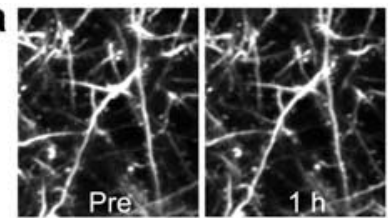

b
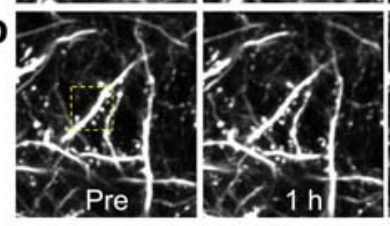

C

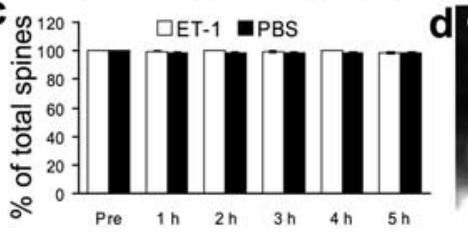

Figure 2. Acute effect of moderate ET-1-induced ischemia on dendritic structure. $\boldsymbol{a}, \boldsymbol{b}$, Injection of PBS (control) ( $(\boldsymbol{a})$ or endothelin $(\boldsymbol{b})$ does not alter dendritic structure monitored during $5 \mathrm{~h}$ of acute imaging. $\boldsymbol{c}$, Relative change in spine number (compared with pretreatment) over 5 h of ET- 1 or PBS treatment ( $p>0.05$ ANOVA; $n=5$ animals for PBS; $n=6$ animals for ET- 1 ). $d$, Image overlay indicates no major changes in spine structure with $5 \mathrm{~h}$ of ET- 1 treatment. Pre, Pretreatment.
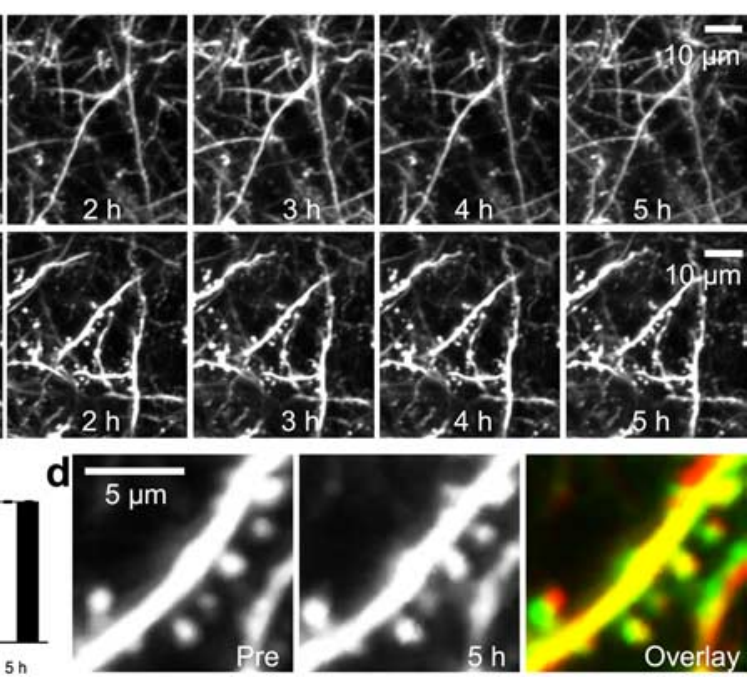

jection drilling and a $52 \pm 9 \%$ reduction in cortical blood flow 15 min after ET-1 injection ( $n=5$ animals). In contrast to the effects of ET-1, PBS vehicle $(n=4$ animals) had no significant effect (Fig. 1b). To measure blood flow locally, we used fluorescent dextran labeling of plasma to observe RBCs as negatively stained structures in a background of labeled plasma (Kleinfeld et al., 1998) (Fig. 1c). Using twophoton imaging of plasma labeled by FITC-dextran, we confirmed that blood flow at the level of capillaries was reduced by endothelin treatment ( $n=3$ animals). Under basal conditions, within the first $100 \mu \mathrm{m}$ of the mouse somatosensory cortex, we find that spines are, on average, $13.0 \pm 0.4 \mu \mathrm{m}(n=250$ spines; $n=3$ animals) from a capillary surface and are supplied by $\sim 100$ RBCs per second. Up to 90 min after endothelin treatment, the RBC supply rate was significantly reduced from $99 \pm 23$ to $64 \pm 19$ RBCs per second at the level of individual capillaries $(p<$ $0.05 ; t$ test; $n=3$ animals) (Fig. $1 d$ ).

number of factors in addition to theoretical optical limits and may not be able to accurately assess submicron changes in spine shape (Oheim et al., 2001). Therefore, we only made a crude assessment of changes in spine length and position using overlays.

The distance between spines and the nearest capillary vessel surface were measured using three-dimensional (3D) image analysis software (Volocity; Improvision, Lexington, MA). Image stacks (100 $\mu \mathrm{m}$ deep) of YFP (dendrite/spines) and Texas Red dextran (capillaries) were obtained in three different animals. A line selection was made between a spine and the surface of the nearest vessel surface using a 3D measurement tool. For statistical analyses, a $t$ test or ANOVA was used, as indicated. Data were expressed as the mean \pm SEM.

\section{Results}

Stroke can be readily modeled in animals using various agents to produce moderate-to-severe levels of ischemia (Watson et al., 1985; Macrae et al., 1993; Biernaskie and Corbett, 2001). We found it necessary to use models that would locally reduce blood flow (as opposed to middle cerebral artery occlusion), to avoid complications associated with collateral circulation commonly found in mice. In addition, the requirement to keep the animal within a stereotaxic frame makes it technically challenging to access carotid arteries that are typically where sutures used to block the middle cerebral artery are placed.

In human stroke, there is typically an ischemic core in which blood flow drops to $<10 \%$ and neuronal cell death is certain (Baron, 2001). Surrounding the core is a penumbra with partial blood flow in which neuroprotective strategies have the best chance of working and in which synaptogenesis may occur (Carmichael, 2003; Katsman et al., 2003). To model the ischemic core, we used RB photoinduced thrombus formation (Watson et al., 1985; Macrae et al., 1993). Endothelin, a vasoconstrictor that produces a moderate drop in blood flow (Fuxe et al., 1992; Biernaskie et al., 2001) was used to model the penumbra. For in vivo two-photon microscopy, a small cranial window was made over the somatosensory cortex. Both models were associated with histologically verified cortical lesions 1-7 d later (Fig. $1 a$ and data not shown).

We first assessed effects of ET-1 on regional blood flow using laser Doppler. These experiments indicated little effect of prein-
After establishing the endothelin ischemia model, we determined its effect on dendritic structure by imaging the apical tuft of layer 5 neurons expressing YFP at $1 \mathrm{~h}$ intervals for $5 \mathrm{~h}$ (Fig. $2 a, b)$. We did not observe any significant time-dependent change in the number of spines over $5 \mathrm{~h}$ under control (PBS; $n=5$ animals) or endothelin injection $(n=6)$ conditions (Fig. $2 c$ ) $(p>0.05$; two-way ANOVA). Spine turnover was minimal for both groups (Fig. $2 c$ ). In control animals, only two spines were lost in four PBS-injected mice in the area sampled $(n=161$ total spines). In ET-1-treated mice, a total of seven spines were lost (from two of six animals), and one new spine appeared of 296 that were evaluated. After ET-1, we made a coarse assessment of changes in spine length and position by using image overlays and observed no major changes (Fig. $2 d$ ). Simultaneous imaging of blood flow and structure confirmed that ET-1-injected animals that were still ischemic at $5 \mathrm{~h}$ had an intact dendritic structure (Fig. 1d). In the example shown, the RBC supply rate was reduced in the right arm of a capillary, and reversals in capillary flow were observed (Fig. 1e,g). The finding that spine structure is retained after up to 5 h of ischemia (Fig. $1 f$ ) suggests that treatment strategies for stroke may be successful within this time period, because basic synaptic architecture and connections appear to be well maintained, perhaps facilitating recovery of function. However, at longer time points ( $>7 \mathrm{~h}$ in $n=2$ animals), endothelin injection produced a pronounced loss of dendritic structure and spines, with spine number being reduced by $60 \%$ at $11 \mathrm{~h}$ without the appearance of any new spines (supplemental Fig. $1 a$, available at www.jneurosci.org as supplemental material).

To contrast the effects of moderate endothelin-induced ischemia, more severe insults were produced using photoactivatable RB to model the ischemic core (Watson et al., 1985). This treatment produced histologically verified lesions at $24 \mathrm{~h}$ (data not shown) and RBC/platelet-derived clots that extended at least 200 $\mu \mathrm{m}$ below the surface, resulting in little or no blood flow and reducing the average $\mathrm{RBC}$ supply rate from $108 \pm 12 \mathrm{RBCs}$ per second for control to $6 \pm 3$ RBCs per second 10 min after photoactivation ( $n=6$ animals quantified; $p<0.05$; $t$ test) (Figs. 
$3 a, b, 4 a)$. See supplemental videos 1 and 2 for images of blood flow in the region shown in Figure 4 and supplemental videos 3 and 4 (videos available at www. jneurosci.org as supplemental material) for a 3D rendering of dendritic and vascular structure under pretreatment conditions and after $30 \mathrm{~min}$ of ischemia (Fig. $3 a, d)$. In YFP-expressing animals injected with FITC or Texas Red dextran (to monitor RBC flow), we examined the relationship between RB-induced ischemia and changes in dendritic structure. We found that 10-40 min after RB-induced ischemia, the normal dendritic structure was completely lost to blebbing (observed in 11 of 13 animals), and virtually no spines were visible (Fig. $3 c, d$, images to $3 \mathrm{~h}$ ). Spines appeared to be lost before any significant loss of capillary wall permeability (assessed by dye loss and signal outside capillaries), indicating that the dendritic damage was not attributed to extravasation (Fig. $3 d$ ). In five animals, the RBinduced clots spontaneously broke up, and reperfusion occurred over a 20-60 min period (confirmed with fluorescent dextran). In three of these animals with confirmed reperfusion (and in a fourth animal that lacked simultaneous bloodflow measurement), we observed that swollen dendrites and absent spines underwent a pronounced restoration in structure during the reperfusion period (Figs. $3 e-i, 4 b-d$ ) (supplemental Fig. 1b, available at www.jneurosci.org as supplemental material). Although many of the spines returned, image overlays indicated that their position or structure was altered (Figs. 3f, 4d) (supplemental Fig. 1b, available at www.jneurosci.org as supplemental material). The ability to observe reversible changes in spine number was dependent on the degree of ischemia because, in 7 of 13 animals, no reperfusion or recovery of dendritic structure was observed (Fig. $3 b, d$ ). In parallel experiments, we observed widespread cortical damage $24 \mathrm{~h}$ after photoactivation, indicating that spines and general dendritic structure that initially recovered eventually degenerated, suggesting that a slower toxic process had been initiated (data not shown). In some animals showing reperfusion, we observed a second phase of clot formation ( $n=3$; $\sim 90$ min later), possibly triggered by clot debris, which leads to irreversible dendritic damage (Fig. $3 e, g-i$ ). In these cases, changes in spine number tracked the changes in blood flow.

In 5 of 18 animals, photoactivation did not lead to clotting or changes in dendritic morphology, suggesting that structural changes were associated with ischemia and not some other effect of RB. Furthermore, control experiments indicated that (1) in the presence of photoactivation (but no RB present), no clotting or change in structure was observed ( $n=2$ animals), and (2) the presence of RB but without photoactivation had no effect $(n=4$ animals).
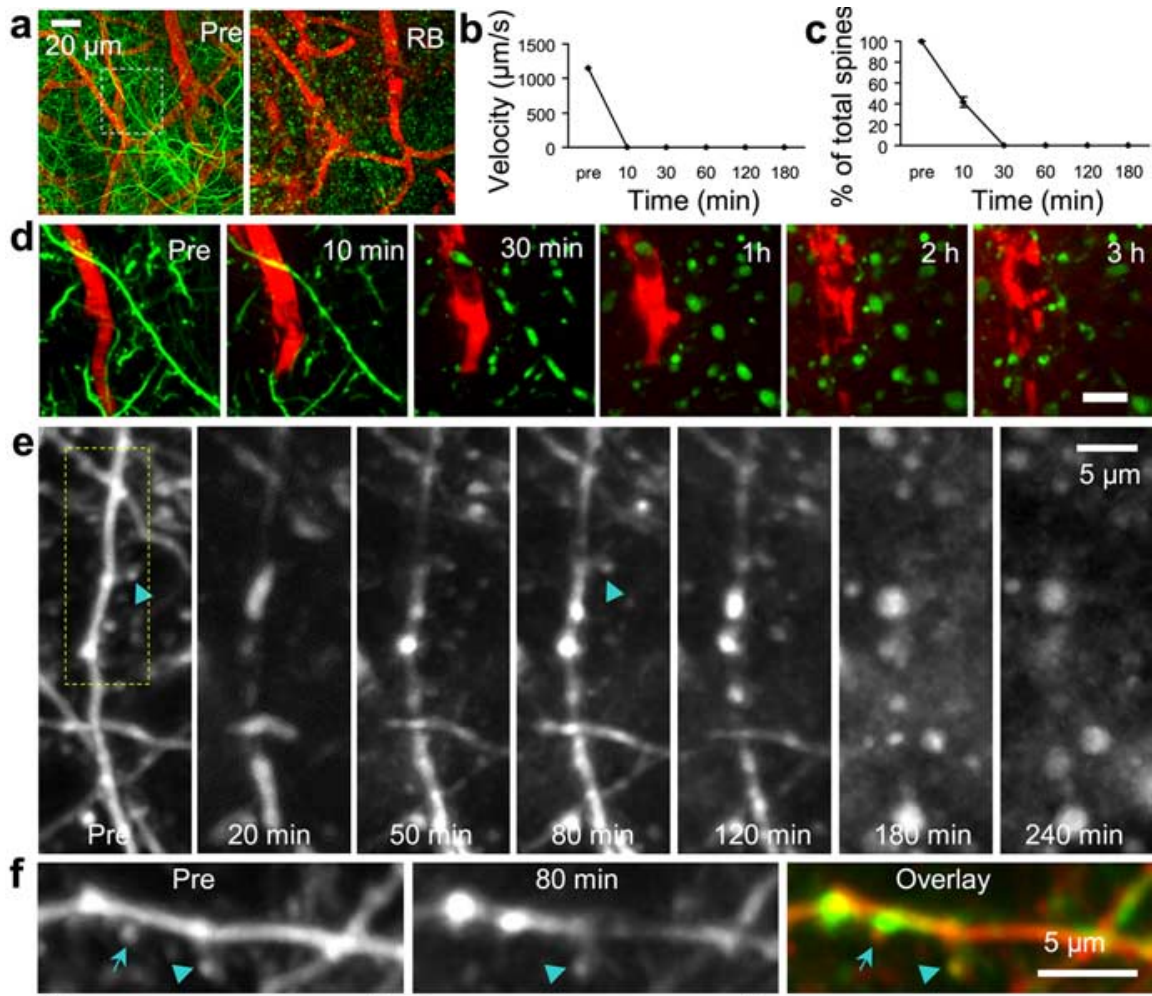

Figure 3. Effect of RB-induced severe ischemia on brain structure. $\boldsymbol{a}$, Images from a YFP mouse showing the vasculature and e, In vivo time-lapse images from another animal showing partial recovery of dendrites and spines with confirmed reperfusion. example of a spine initially lost that recovered with reperfusion. $f$, A dendritic region from the yellow box recovered at the same place. The arrow shows a spine not recovered after reperfusion. Quantification of spine number $(\boldsymbol{g})$, blood-flow velocity $(\boldsymbol{h})$, and flux for this animal (i). Pre, Pretreatment.

\section{Discussion}

Our results indicate that moderate ischemia (ET-1 penumbra model) is associated with spine stability over the first $5 \mathrm{~h}$, indicating that, if stroke treatment was performed within this time window, synapse structure would be relatively preserved. However, the pronounced loss and gradual degeneration of dendritic structure and spines at longer time points $(>7 \mathrm{~h}$ ) (supplemental Fig. $1 a$, available at www.jneurosci.org as supplemental material) are consistent with ischemia, activating signal transduction cascades that lead to apoptosis or necrosis (Liang et al., 2004).

Previous studies in cultured brain slices prepared from 7-dold rat pups suggest that the number of spines and filopodia can be increased by $10 \mathrm{~min}$ of transient ischemia (Piccini and Malinow, 2001; Jourdain et al., 2002). In our in vivo study, it was not possible to produce such short-term ischemia. However, with moderate endothelin-induced ischemia, we did not observe any significant increase in spine or filopodia number indicating differences between the models used in vitro with regard to developmental maturation or other factors. 


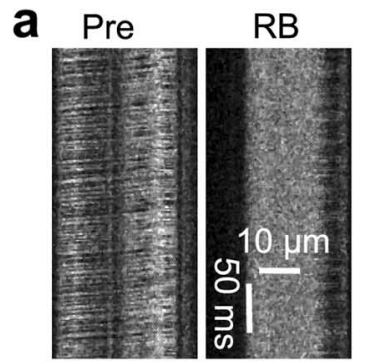

$2814.6 \mu \mathrm{m} / \mathrm{s} 0 \mu \mathrm{m} / \mathrm{s}$ $209 \mathrm{RBC} / \mathrm{s} \quad 0 \mathrm{RBC} / \mathrm{s}$
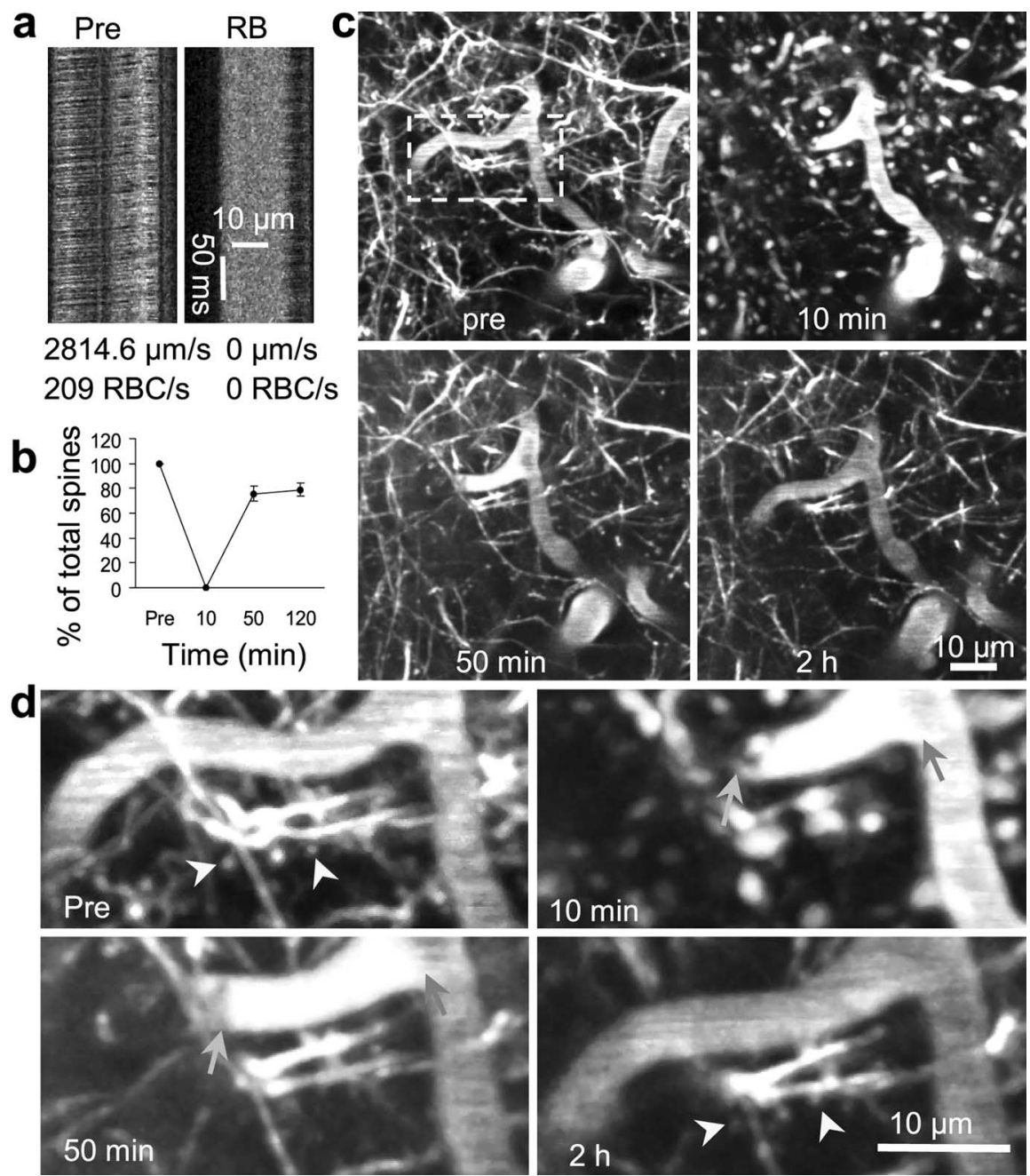

Figure 4. Spine loss and recovery with severe transient ischemia and reperfusion. $\boldsymbol{a}$, Line scan through a section of capillary shown in $\mathbf{c}$ and $\boldsymbol{d}$ to monitor blood flow. Blood flow stops 10 min after photoactivation of RB. $\boldsymbol{b}$, Spine number expressed as percentage of pre-RB photoactivation (average from 5 regions). c, In vivo images of a dendritic region showing beading and recovery with reperfusion (perfusion can be assessed by the striped image of the capillary that reflects moving RBCs). $\boldsymbol{d}$, Highermagnification view of the boxed region in c showing spine loss with ischemia and recovery with reperfusion. Both the dendrites (YFP) and the vessels (FITC- dextran) were labeled with the same color. Arrowheads show examples of spines initially lost and recovered with reperfusion. Arrows indicate RB-induced clotting in a section of a capillary that resolved spontaneously over a $2 \mathrm{~h}$ period after photoactivation. Pre, Pretreatment.

In the case of severe ischemia in vivo ( $\mathrm{RB}$ model), spines and dendrites were rapidly damaged and became unrecognizably swollen within 10-30 min. A similar morphology has been observed with brain slices during glutamate-induced excitotoxicity (Jarvis et al., 1999). The cytoskeleton appears to be quite resilient in vivo, because reperfusion is associated with a remarkable reversal of swollen spines and their dendrites toward normal structure, consistent with work done in vitro (Hasbani et al., 2001; Kirov et al., 2004). In the case of ion imbalance brought about by brain slicing in vitro, a similar phenomenon occurs, and the recovered dendrites are apparently functional because synaptic transmission is observed (Hasbani et al., 2001; Kirov et al., 2004). These in vitro findings suggest that the presynaptic terminal is still attached during the reversible dendritic swelling. From these studies, we believe that spines are not visible during the severe ischemia, because the dendrites are severely swollen and beaded, thus occluding them. Alternatively, after ischemic cytoskeletal breakdown, spines may be like flaccid skin without bones and appear invisible because their volume is greatly reduced.
These data offer hope that if robust neuroprotective strategies were applied within $5 \mathrm{~h}$ of stroke onset, the prognosis for recovery (of moderately ischemic penumbra regions) would be good, because the basic excitatory wiring diagram and associated dendritic spines are mostly intact. In the case of severe ischemia, dendritic structure can partially recover if reperfusion stimulated by agents such as tissue plasminogen activator occurs within 20-60 min. Interestingly, recent studies indicate that tissue plasminogen activator may even promote spine motility (Oray et al., 2004).

\section{References}

Barber PA, Demchuk AM, Hirt L, Buchan AM (2003) Biochemistry of ischemic stroke. Adv Neurol 92:151-164.

Baron JC (2001) Perfusion thresholds in human cerebral ischemia: historical perspective and therapeutic implications. Cerebrovasc Dis 11 [Suppl 1]:2-8.

Biernaskie J, Corbett D (2001) Enriched rehabilitative training promotes improved forelimb motor function and enhanced dendritic growth after focal ischemic injury. J Neurosci 21:5272-5280.

Biernaskie J, Corbett D, Peeling J, Wells J, Lei H (2001) A serial MR study of cerebral blood flow changes and lesion development following endothelin-1-induced ischemia in rats. Magn Reson Med 46:827-830.

Bonhoeffer T, Yuste R (2002) Spine motility. Phenomenology, mechanisms, and function. Neuron 35:1019-1027.

Carmichael ST (2003) Plasticity of cortical projections after stroke. The Neuroscientist 9:64-75.

Feng G, Mellor RH, Bernstein M, Keller-Peck C, Nguyen QT, Wallace M, Nerbonne JM, Lichtman JW, Sanes JR (2000) Imaging neuronal subsets in transgenic mice expressing multiple spectral variants of GFP. Neuron 28:41-51.

Fuxe K, Kurosawa N, Cintra A, Hallstrom A, Goiny M, Rosen L, Agnati LF, Ungerstedt U (1992) Involvement of local ischemia in endothelin-1 induced lesions of the neostriatum of the anaesthetized rat. Exp Brain Res 88:131-139.

Grutzendler J, Kasthuri N, Gan WB (2002) Longterm dendritic spine stability in the adult cortex. Nature 420:812-816.

Hasbani MJ, Schlief ML, Fisher DA, Goldberg MP (2001) Dendritic spines lost during glutamate receptor activation reemerge at original sites of synaptic contact. J Neurosci 21:2393-2403.

Hering H, Sheng M (2001) Dendritic spines: structure, dynamics and regulation. Nat Rev Neurosci 2:880-888.

Holtmaat AJ, Trachtenberg JT, Wilbrecht L, Shepherd GM, Zhang X, Knott GW, Svoboda K (2005) Transient and persistent dendritic spines in the neocortex in vivo. Neuron 45:279-291.

Jarvis CR, Lilge L, Vipond GJ, Andrew RD (1999) Interpretation of intrinsic optical signals and calcein fluorescence during acute excitotoxic insult in the hippocampal slice. NeuroImage 10:357-372.

Jourdain P, Nikonenko I, Alberi S, Muller D (2002) Remodeling of hippocampal synaptic networks by a brief anoxia-hypoglycemia. J Neurosci 22:3108-3116.

Katsman D, Zheng J, Spinelli K, Carmichael ST (2003) Tissue microenvironments within functional cortical subdivisions adjacent to focal stroke. J Cereb Blood Flow Metab 23:997-1009.

Kirov SA, Petrak LJ, Fiala JC, Harris KM (2004) Dendritic spines disappear with chilling but proliferate excessively upon rewarming of mature hippocampus. Neuroscience 127:69-80. 
Kleinfeld D, Denk W (2000) Imaging neurons: a laboratory manual. Cold Spring Harbor, NY: Cold Spring Harbor Laboratory.

Kleinfeld D, Mitra PP, Helmchen F, Denk W (1998) Fluctuations and stimulus-induced changes in blood flow observed in individual capillaries in layers 2 through 4 of rat neocortex. Proc Natl Acad Sci USA 95:15741-15746.

Kolb B, Gibb R, Gorny G (2003) Experience-dependent changes in dendritic arbor and spine density in neocortex vary qualitatively with age and sex. Neurobiol Learn Mem 79:1-10.

Liang D, Dawson TM, Dawson VL (2004) What have genetically engineered mice taught us about ischemic injury? Curr Mol Med 4:207-225.

Macrae IM, Robinson MJ, Graham DI, Reid JL, McCulloch J (1993) Endothelin-1-induced reductions in cerebral blood flow: dose dependency, time course, and neuropathological consequences. J Cereb Blood Flow Metab 13:276-284.
Oheim M, Beaurepaire E, Chaigneau E, Mertz J, Charpak S (2001) Twophoton microscopy in brain tissue: parameters influencing the imaging depth. J Neurosci Methods 111:29-37.

Oray S, Majewska A, Sur M (2004) Dendritic spine dynamics are regulated by monocular deprivation and extracellular matrix degradation. Neuron 44:1021-1030.

Piccini A, Malinow R (2001) Transient oxygen-glucose deprivation induces rapid morphological changes in rat hippocampal dendrites. Neuropharmacology 41:724-729.

Trachtenberg JT, Chen BE, Knott GW, Feng G, Sanes JR, Welker E, Svoboda $\mathrm{K}$ (2002) Long-term in vivo imaging of experience-dependent synaptic plasticity in adult cortex. Nature 420:788-794.

Watson BD, Dietrich WD, Busto R, Wachtel MS, Ginsberg MD (1985) Induction of reproducible brain infarction by photochemically initiated thrombosis. Ann Neurol 17:497-504. 